九州大学学術情報リポジトリ

Kyushu University Institutional Repository

\title{
Dual structures of chaos and turbulence, and their dynamic scaling laws
}

Mori, Hazime

Research Institute for Applied Mechanics, Kyushu University

Okamura, Makoto

Research Institute for Applied Mechanics, Kyushu University

http://hdl. handle. net/2324/1910480

出版情報: Physical Review E. 80 (5)，pp.051124-1-051124-7，2009-11-24. American Physical Society

バージョン :

権利関係 : ๑2009 American Physical Society 


\title{
Dual structures of chaos and turbulence, and their dynamic scaling laws
}

\author{
Hazime Mori and Makoto Okamura* \\ Research Institute for Applied Mechanics, Kyushu University, Kasuga 816-8580, Japan
}

(Received 1 July 2009; published 24 November 2009)

\begin{abstract}
The decay form of the time correlation function $U_{n}(t)$ of a state variable $u_{n}(t)$ with a small wave number $k_{n}$ has been shown to take the algebraic decay $1 /\left\{1+\left(\gamma_{n a} t\right)^{2}\right\}$ in the initial regime $t<\tau_{n}^{(\gamma)}$ and the exponential decay $\alpha_{n e} \exp \left(-\gamma_{n e} t\right)$ in the final regime $t>\tau_{n}^{(\gamma)}$, where $\tau_{n}^{(\gamma)}$ denotes the decay time of the memory function $\Gamma_{n}(t)$. This dual structure of $U_{n}(t)$ is generated by the deterministic short orbits in the initial regime and the stochastic long orbits in the final regime, thus giving the outstanding features of chaos and turbulence. The $k_{n}$ dependence of $\gamma_{n a}, \alpha_{n e}$, and $\gamma_{n e}$ is obtained for the chaotic Kuramoto-Sivashinsky equation, and it is shown that if $k_{n}$ is sufficiently small, then the dual structure of $U_{n}(t)$ obeys a hydrodynamic scaling law in the final regime $t>\tau_{n}^{(\gamma)}$ with scaling exponent $z=2$ and a dynamic scaling law in the initial regime $t<\tau_{n}^{(\gamma)}$ with scaling exponent $z=1$. If $k_{n}$ is increased so that the decay time $\tau_{n}^{(u)}$ of $U_{n}(t)$ becomes equal to the decay time $\tau_{n}^{(\gamma)}$, then the decay form of $U_{n}(t)$ becomes the power-law decay $t^{-3 / 2}$ in the final regime.
\end{abstract}

DOI: 10.1103/PhysRevE.80.051124

PACS number(s): 05.40.-a, 05.45.-a, 05.10.Gg, 02.50.Ey

\section{INTRODUCTION}

Chaos and turbulence consist of unstable nonperiodic orbits with positive Liapunov exponents, and their sensitive dependence on the initial points brings about an exponential increase of the initial errors, so that the nonperiodic orbits become stochastic and random after an initial regime [1-3]. Therefore, the nonperiodic orbits in the time correlation function $U_{n}(t)$ of a state variable $u_{n}(t)$ consist of deterministic short orbits in an initial regime $t<\tau_{n}^{(\gamma)}$ and stochastic long orbits in a final regime $t>\tau_{n}^{(\gamma)}$, where $\tau_{n}^{(\gamma)}$ is the decay time of the memory function $\Gamma_{n}(t)$. This leads to a dual structure of the randomization of the nonperiodic orbits in $U_{n}(t)$, which is governed by a non-Markovian evolution equation with the memory function $\Gamma_{n}(t)$ as the kernel of convolution $[4,5]$.

Indeed it has been shown for the Kuramoto-Sivashinsky (KS) equation in a previous paper [4] that such a dual structure is displayed by the time correlation function $U_{n}(t)$ and its memory function $\Gamma_{n}(t)$ for a small wave number $k_{n}$. Namely, if the decay time $\tau_{n}^{(u)}$ of $U_{n}(t)$ is sufficiently larger than the decay time $\tau_{n}^{(\gamma)}$ of the memory function $\Gamma_{n}(t)$, then the decay form of $U_{n}(t)$ consists of the algebraic decay $1 /\left\{1+\left(\gamma_{n a} t\right)^{2}\right\}$ in the initial regime $t<\tau_{n}^{(\gamma)}$ and the exponential decay $\alpha_{n e} \exp \left(-\gamma_{n e} t\right)$ in the final regime $t>\tau_{n}^{(\gamma)}$ [4], leading to an outstanding dual structure of chaos and turbulence.

If the wave number $k_{n}$ is increased, then $\tau_{n}^{(u)}$ decreases and becomes of the same order of magnitude as $\tau_{n}^{(\gamma)}$. Then, the exponential decay is replaced by a slower decay. Indeed a power-law decay $t^{-3 / 2}$ appears in the final regime $t>\tau_{n}^{(\gamma)}[5]$. The algebraic decay in the initial regime $t<\tau_{n}^{(\gamma)}$ still holds, leading to a new dual structure of chaos and turbulence.

The purposes of the present paper are to study the time correlation function $U_{n}(t)$ in the statistically steady state of chaos and turbulence and to clarify the dual structures of the decay form of $U_{n}(t)$ due to the randomization of the nonperiodic orbits. We first obtain the $k_{n}$ dependence of the basic

\footnotetext{
*okamura@ riam.kyushu-u.ac.jp
}

parameters $\gamma_{n e}, \gamma_{n a}$, and $\alpha_{n e}$ explicitly for the chaotic KS equation $[4,6,7]$ and then derive dynamic scaling laws for the dual structures of the power spectra of $u_{n}(t)$.

The present paper is organized as follows. In Sec. II, we introduce the memory function $\Gamma_{n}(t)$ and the dual structure of the decay form of $U_{n}(t)$ for the chaotic KS equation with $\tau_{n}^{(\gamma)} \ll \tau_{n}^{(u)}$. In Sec. III, we consider the case where $k_{n}$ is very small so that $\tau_{n}^{(\gamma)} \ll \tau_{n}^{(u)}$. Then we have the exponential decay in the final regime $t>\tau_{n}^{(\gamma)}$, which obeys a hydrodynamic scaling law with scaling exponent $z=2$. In Sec. IV, we derive the algebraic decay in the initial regime $t<\tau_{n}^{(\gamma)}$ from the similarity approximation (SA) equation for $U_{n}(t)$. This decay is shown to obey a dynamic scaling law with scaling exponent $z=1$. In Sec. $\mathrm{V}$, we discuss the case where $\tau_{n}^{(\gamma)} \approx \tau_{n}^{(u)}$. Then we obtain the power-law decay $t^{-3 / 2}$ in the final regime for the chaotic KS equation. In Sec. VI, we discuss three structures of the power spectra that correspond to the three types of decay forms: the exponential decay, the algebraic decay, and the power-law decay. Section VII is devoted to a summary.

\section{DECAY FORMS OF $U_{n}(t)$ IN CASE OF $\tau_{n}^{(\gamma)} \ll \tau_{n}^{(u)}$}

We treat the chaotic KS equation for the state variable $u(x, t)$,

$$
u_{t}+u u_{x}+u_{x x}+u_{x x x x}=0
$$

under the periodic boundary condition $u(x, t)=u(x+L, t)$ with $L=500[4,7]$. The Fourier transform

$$
u_{n}(t) \equiv \int_{0}^{L} u(x, t) e^{-i k_{n} x} d x \quad(n=0, \pm 1, \ldots, \pm N)
$$

with wave number $k_{n}=2 \pi n / L$ and $N=256$ yields $N$ evolution equations

$$
d u_{n}(t) / d t=\left(k_{n}^{2}-k_{n}^{4}\right) u_{n}(t)+N_{n}(t),
$$

where $N_{n}(t)$ is a nonlinear inertial force given by 


$$
N_{n}(t) \equiv-\frac{i}{L} \sum_{m=-N}^{N} k_{m} u_{n-m}(t) u_{m}(t)
$$

The state variable $u_{n}(t)$ fluctuates with time according to the chaotic evolution equations (2.3), leading to a statistically steady state [7]. This fluctuation $u_{n}(t)$ gives the simplest example of the turbulent hydrodynamic fluctuations.

Now let us consider the time evolution of the time correlation function

$$
U_{n}(t) \equiv\left\langle u_{n}(t) u_{n}^{*}(0)\right\rangle /\left\langle\left|u_{n}(0)\right|^{2}\right\rangle,
$$

where the angular brackets denote the long-time average,

$$
\left\langle u_{n}(t) u_{n}^{*}(0)\right\rangle \equiv \frac{1}{M} \sum_{j=0}^{M-1} u_{n}\left(t+T_{0}+40 j\right) u_{n}^{*}\left(T_{0}+40 j\right),
$$

with $M=\left(T-T_{0}\right) / 40=1.2 \times 10^{6}, T=5 \times 10^{7}, \quad T_{0}=10^{3} \quad$ [4]. Then, as shown in previous papers $[4,7]$, the projection operator formalism leads to a non-Markovian evolution equation of the form

$$
\frac{d}{d t} U_{n}(t)=-\int_{0}^{t} \Gamma_{n}(s) U_{n}(t-s) d s
$$

where $\Gamma_{n}(t)$ is the memory function given by the second fluctuation-dissipation theorem [8-11],

$$
\Gamma_{n}(t)=\left\langle r_{n}(t) r_{n}^{*}(0)\right\rangle /\left\langle\left|u_{n}(0)\right|^{2}\right\rangle
$$

in terms of the fluctuating forces $r_{n}(t)$ [4,7]. Taking the Fourier-Laplace transform of Eq. (2.7), we obtain the time correlation spectra

$$
U_{n}(\omega) \equiv \int_{0}^{\infty} U_{n}(t) e^{-i \omega t} d t=\frac{1}{i \omega+\Gamma_{n}(\omega)}
$$

in terms of the memory spectra

$$
\Gamma_{n}(\omega) \equiv \int_{0}^{\infty} \Gamma_{n}(t) e^{-i \omega t} d t=\Gamma_{n}^{\prime}(\omega)+i \Gamma_{n}^{\prime \prime}(\omega),
$$

where we have $\Gamma_{n}(\omega=0)=1 / U_{n}(\omega=0)$. These memory spectra $\Gamma_{n}(\omega)$ describe the transport processes, such as the chaosinduced friction and turbulent viscosity $[4,8,9]$. Therefore, the hydrodynamical motions and associated transport processes can be described by these time correlation spectra $U_{n}(\omega)$ and memory spectra $\Gamma_{n}(\omega)$.

As the basic time scales of the system (2.3), let us introduce the following integral time scales of $U_{n}(t)$ and $\Gamma_{n}(t)$ [12]:

$$
\begin{gathered}
\tau_{n}^{(u)} \equiv \int_{0}^{\infty} U_{n}(t) d t=U_{n}(\omega=0), \\
\tau_{n}^{(\gamma)} \equiv \frac{1}{\Gamma_{n}(t=0)} \int_{0}^{\infty} \Gamma_{n}(t) d t=\frac{1}{\Gamma_{n}(t=0)} \frac{1}{\tau_{n}^{(u)}}
\end{gathered}
$$

for the KS equation, where Eqs. (2.9) and (2.12) lead to

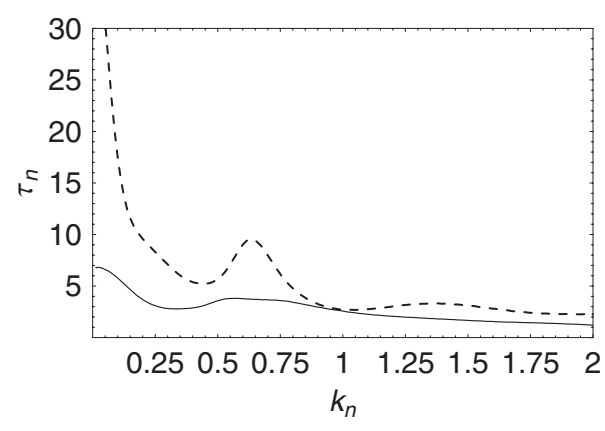

FIG. 1. The time scales $\tau_{n}^{(u)}$ (broken line) and $\tau_{n}^{(\gamma)}$ (solid line) as functions of $k_{n}$. This indicates $\tau_{n}^{(\gamma)}<\tau_{n}^{(u)}$.

$$
\begin{gathered}
\Gamma_{n}(\omega=0)=\frac{1}{U_{n}(\omega=0)}=\frac{1}{\tau_{n}^{(u)}}, \\
\Gamma_{n}(t=0)=\frac{1}{\tau_{n}^{(u)} \tau_{n}^{(\gamma)}} .
\end{gathered}
$$

Therefore, both of $\tau_{n}^{(u)}$ and $\tau_{n}^{(\gamma)}$ can be given by the memory spectra $\Gamma_{n}(\omega)$.

Figure 1 shows the dependence of the integral time scales $\tau_{n}^{(u)}$ and $\tau_{n}^{(\gamma)}$ on the wave number $k_{n}$, which is obtained by the direct numerical simulation (DNS) of Eqs. (2.5), (2.11), and (2.12). This indicates that

$$
\widetilde{\tau}_{n} \equiv \tau_{n}^{(\gamma)} / \tau_{n}^{(u)} \ll 1 \quad \text { for } k_{n}<0.15 \quad(n<11) .
$$

Namely, if the wave number $k_{n}$ is sufficiently small, then the integral time scale $\tau_{n}^{(\gamma)}$ of $\Gamma_{n}(t)$ becomes much smaller than the integral time scale $\tau_{n}^{(u)}$ of $U_{n}(t)$. Then, as shown by the DNS of Eqs. (2.3) and (2.5) in the previous paper [4], the decay form of $U_{n}(t)$ is given by the algebraic decay

$$
U_{n}(t)=\frac{1}{1+\left(\gamma_{n a} t\right)^{2}}
$$

in the initial regime $t<\tau_{n}^{(\gamma)}$ and the exponential decay

$$
U_{n}(t)=\alpha_{n e} \exp \left(-\gamma_{n e} t\right)
$$

in the final regime $t>\tau_{n}^{(\gamma)}$, where we have

$$
\begin{gathered}
\gamma_{n e}=1 / \tau_{n}^{(u)}, \\
\gamma_{n a}=1 /\left(2 \tau_{n}^{(u)} \tau_{n}^{(\gamma)}\right)^{1 / 2}=\gamma_{n e} /\left(2 \widetilde{\tau}_{n}\right)^{1 / 2} .
\end{gathered}
$$

Here Eq. (2.19) will be derived from Eqs. (4.8) and (4.9) in Sec. IV.

Figure 2 clearly shows that the DNS of $U_{n}(t)$ consists of the two decay forms: the algebraic decay (2.16) and the exponential decay (2.17). The orbits in the long-time average (2.5) consist of the deterministic short orbits in the initial regime $t<\tau_{n}^{(\gamma)}$ and the stochastic long orbits with positive Liapunov exponents in the final regime $t>\tau_{n}^{(\gamma)}$. The algebraic decay (2.16) in the initial regime $t<\tau_{n}^{(\gamma)}$ is reversible under the time reversal and produced by the reversible dynamical coherence of the deterministic short orbits, as will be shown in Sec. IV, whereas the irreversible exponential decay (2.17) in the final regime $t>\tau_{n}^{(\gamma)}$ is generated by the decay of the 


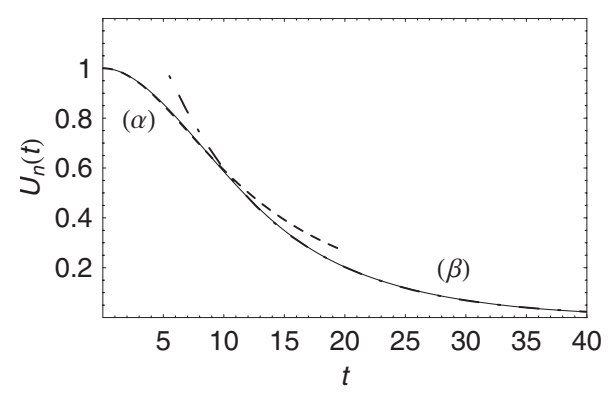

FIG. 2. A dual structure of $U_{n}(t)$ for $k_{n}=0.13(n=10)$ : $(\alpha)--$ - , the algebraic decay (2.16); $(\beta)-\cdot-$, the exponential decay (2.17); and -, DNS, where $\tau_{n}^{(\gamma)}=5.2$.

time correlation due to the randomization and mixing of the stochastic long orbits. Here a typical randomization is provided by the chaos-induced friction and the turbulent viscosity. Thus it turns out that the time correlation function $U_{n}(t)$ of chaos and turbulence consists of the dual structure of the algebraic decay (2.16) and the exponential decay (2.17). Here it should be noted that such a dual structure is possible only for the non-Markovian evolution equation such as Eq. (2.7), hence giving the outstanding features of chaos and turbulence. A simple physical picture for this dual structure of $U_{n}(t)$ is summarized in Table I. A theoretical derivation of the decay forms [Eqs. (2.16) and (2.17)] will be given in Secs. III and IV.

\section{EXPONENTIAL DECAY (2.17) AND ITS HYDRODYNAMIC SCALING LAW FOR LARGE-DISTANCE AND LONG-TIME BEHAVIORS}

In this section, we assume that the wave numbers $k_{n}$ are sufficiently small so that Eq. (2.15) holds: $\tau_{n}^{(\gamma)} \ll \tau_{n}^{(u)}$. Then the memory function $\Gamma_{n}(t)$ decays to zero immediately in the time scale of $\tau_{n}^{(u)}$, so that we may assume the asymptotic estimation

$$
\Gamma_{n}(t)=2 \gamma_{n e} \delta(t),
$$

where $\delta(t)$ is the Dirac delta function of $t$. Inserting Eq. (3.1) into Eq. (2.10), we obtain the decay rate

$$
\Gamma_{n}(\omega)=\gamma_{n e}=1 / \tau_{n}^{(u)},
$$

where Eq. (2.13) has been used. In the following, let us consider the time evolution of $U_{n}(t)$ in the final regime $t>\tau_{n}^{(\gamma)}$. Then inserting Eq. (3.1) into Eq. (2.7), we have

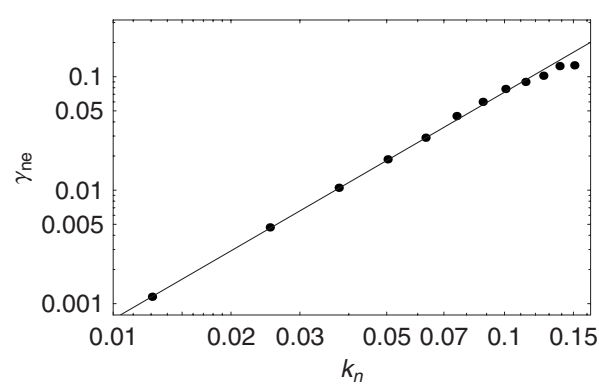

FIG. 3. The $k_{n}$ dependence of $\gamma_{n e}$ with the solid line, showing Eq. (3.5).

$$
d U_{n}(t) / d t=-\gamma_{n e} U_{n}(t),
$$

which is integrated to give

$$
U_{n}(t)=\alpha_{n e} \exp \left(-\gamma_{n e} t\right) \text { for } t>\tau_{n}^{(\gamma)} .
$$

This equation justifies the exponential decay (2.17) under condition (2.15). It should be noted here that Eq. (2.7) is reversible under the time reversal, but Eq. (3.1) breaks the time-reversal symmetry, leading to the irreversibility of the exponential decay (3.4). It should also be noted that the initial regime $t<\tau_{n}^{(\gamma)}$, where the algebraic decay (2.16) holds, is shrunk to zero by assuming Eq. (3.1). In Sec. IV, we shall remove this restriction which neglects the structure of the initial regime.

The dynamic structures of chaos and turbulence are built up by the irreversible exponential decay (3.4) in the final regime $t>\tau_{n}^{(\gamma)}$. In order to obtain the dynamic structures explicitly, let us determine the $k_{n}$ dependence of $\gamma_{n e}$ and $\alpha_{n e}$ in Eq. (3.4) explicitly. As shown in Fig. 3, the decay rate $\gamma_{n e}$ depends on $k_{n}$ as

$$
\gamma_{n e}=\lambda k_{n}^{z} \quad(\lambda=7.3, \quad z=2)
$$

for $k_{n}<0.1$. In order to see the universality of the exponential decay (3.4) for small wave numbers $k_{n}$, let us consider its spectrum

$$
\begin{gathered}
U_{n e}(\omega) \equiv \alpha_{n e} \int_{0}^{\infty} \exp \left(-\gamma_{n e} t-i \omega t\right) d t \\
=k_{n}^{-z} \Phi_{n}\left(\omega / k_{n}^{z}\right),
\end{gathered}
$$

where, writing as $\eta \equiv \omega / k_{n}^{z}, \zeta \equiv k_{n}^{z} t$, we have

TABLE I. A physical picture for the decay form of $U_{n}(t)$ in case of $\tau_{n}^{(\gamma)} \ll \tau_{n}^{(u)}$. Nonperiodic orbits of chaos and turbulence have positive Liapunov exponents and become stochastic and random in a time scale $\tau_{n}^{(\gamma)}$, hence producing the outstanding dual structures for the decay forms of $U_{n}(t)$.

\begin{tabular}{ll}
\hline \multicolumn{1}{c}{ Initial regime $t<\tau_{n}^{(\gamma)}$} & \multicolumn{1}{c}{ Final regime $t>\tau_{n}^{(\gamma)}$} \\
\hline Its main orbits are the deterministic short orbits. & Its main orbits are the stochastic long orbits. \\
The dynamical coherence of these orbits & The randomization and mixing of these orbits \\
produces an algebraic decay $(2.16):$ & generate an exponential decay $(2.17):$ \\
$U_{n}(t)=1 /\left\{1+\left(\gamma_{n a} t\right)^{2}\right\}$. & $U_{n}(t)=\alpha_{n e} \exp \left(-\gamma_{n e} t\right)$. \\
$\gamma_{n a}=1 /\left\{\left(2 \widetilde{\tau}_{n}\right)^{1 / 2} \tau_{n}^{(u)}\right\}=\mu k_{n}(\mu=0.65)$. & $\gamma_{n e}=1 / \tau_{n}^{(u)}=\lambda k_{n}^{2}(\lambda=7.3)$. \\
\hline \hline
\end{tabular}




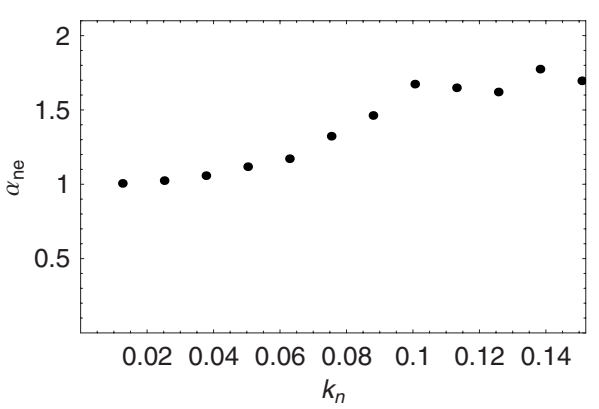

FIG. 4. The $k_{n}$ dependence of $\alpha_{n e}$, showing $\alpha_{n e} \approx 1$ for $k_{n}$ $<0.04$.

$$
\Phi_{n}(\eta) \equiv \alpha_{n e} \int_{0}^{\infty} e^{-\lambda \zeta-i \eta \zeta} d \zeta=\frac{\alpha_{n e}}{\lambda+i \eta} .
$$

As shown in Fig. 4, the parameter $\alpha_{n e}$ depends on $k_{n}$, but we can put $\alpha_{n e} \approx 1$ for $k_{n}<0.04$. Then $\Phi_{n}(\eta)$ becomes independent of $k_{n}$, so that $U_{n e}(\omega)$ in Eq. (3.7) gives a hydrodynamic scaling law with scaling exponent $z=2$. However, this scaling law is different from the dynamic scaling law proposed by Forster-Nelson-Stephen for the Burgers turbulence, whose scaling exponent is $z=3 / 2$ [13-16]. Although a deviation of $z$ from 2 could be possible when $L$ in Eq. (2.2) becomes larger, there do not exist any DNS and experiments that imply $z=3 / 2[6,15,16]$.

Using Eq. (2.9), we can define the memory spectrum $\Gamma_{n}(\omega)$ by

$$
\Gamma_{n}(\omega)=-i \omega+\frac{1}{U_{n}(\omega)} .
$$

Therefore, the memory spectrum of the exponential decay (3.4) is given by inserting Eq. (3.7) into $U_{n}(\omega)$ in Eq. (3.9), i.e.,

$$
\Gamma_{n e}(\omega)=k_{n}^{z} \Psi_{n}\left(\omega / k_{n}^{z}\right),
$$

where, using Eq. (3.8), we have defined

$$
\Psi_{n}(\eta) \equiv-i \eta+\frac{1}{\Phi_{n}(\eta)}=\frac{\lambda}{\alpha_{n e}}-i\left(1-\frac{1}{\alpha_{n e}}\right) \eta
$$

The turbulent viscosity $[8,13,17]$ is given by

$$
\nu_{T}\left(k_{n}, \omega\right) \equiv k_{n}^{-2} \Gamma_{n e}(\omega)=\Psi_{n}\left(\omega / k_{n}^{z}\right) .
$$

Now let us assume that $k_{n}<0.04$, i.e., $n \leq 3$, so that Fig. 4 leads to $\alpha_{n e} \approx 1$. Then, in the final regime $\omega<\lambda k_{n}^{z}$, Eqs. (3.7), (3.10), and (3.12) lead to the spectra

$$
\begin{gathered}
U_{n e}(\omega)=k_{n}^{-z} \frac{\lambda-i \omega / k_{n}^{z}}{\lambda^{2}+\left(\omega / k_{n}^{z}\right)^{2}}, \\
\Gamma_{n e}(\omega)=\lambda k_{n}^{z}, \\
\nu_{T}\left(k_{n}, \omega\right)=\lambda,
\end{gathered}
$$

where $\Gamma_{n e}(\omega=0)=\gamma_{n e}=1 / U_{n e}(\omega=0)$. These spectra give a hydrodynamic approximation to the time correlation spectrum $U_{n}(\omega)$ and the memory spectrum $\Gamma_{n}(\omega)$, satisfying the
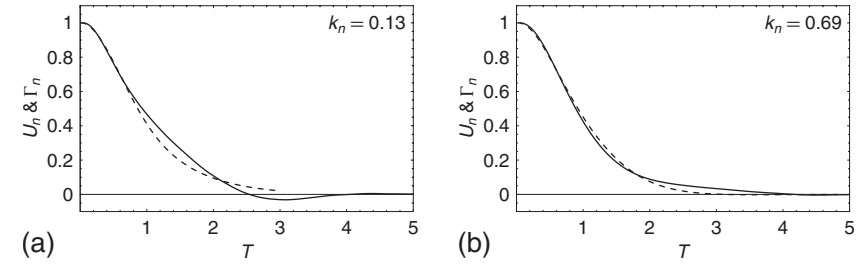

FIG. 5. Numerical verification of the similarity between $U_{n}(t)$ (broken line) and $\Gamma_{n}(t)$ (solid line) in the initial regime $T<\widetilde{\tau}_{n}$.

hydrodynamic scaling law with scaling exponent $z=2$ in the large-distance and long-time regime with $k_{n}<0.04$ and $\omega$ $<\lambda k_{n}^{2}$. Thus it turns out that the hydrodynamic structures [Eqs. (3.13)-(3.15)], produced by the irreversible randomization of the stochastic long orbits in the final regime $t>\tau_{n}^{(\gamma)}$, obey a hydrodynamic scaling law with scaling exponent $z$ $=2$.

\section{ALGEBRAIC DECAY (2.16) AND ITS DYNAMIC SCALING LAW}

In order to derive the algebraic decay (2.16) theoretically, let us consider the closure of the evolution equation (2.7) for $U_{n}(t)$ in the initial regime $t<\tau_{n}^{(\gamma)}$ from the viewpoint of the similarity between $U_{n}(t)$ and $\Gamma_{n}(t)$ pointed out in previous papers [4,5]. Namely, instead of assuming Eqs. (3.1) and (2.15), we consider the decay form of $\Gamma_{n}(t)$ explicitly according to Eq. (3.9) with wave numbers $k_{n}$ and frequencies $\omega$ from zero to $\pm \infty$.

Figure 5 shows that the decay form of $\Gamma_{n}(t)$ is similar to that of $U_{n}(t)$ in the initial regime $T / \widetilde{\tau}_{n}=t / \tau_{n}^{(\gamma)}<1$. This similarity leads to a closure of the evolution equation (2.7) in the initial regime $t<\tau_{n}^{(\gamma)}$, which enables us to derive the algebraic decay (2.16) theoretically.

Let us normalize $t$ and $\tau_{n}^{(\gamma)}$ by $\tau_{n}^{(u)}$ as

$$
T=t / \tau_{n}^{(u)}, \quad \widetilde{\tau}_{n}=\tau_{n}^{(\gamma)} / \tau_{n}^{(u)},
$$

where Fig. 1 indicates $\tilde{\tau}_{n} \leq 1$. Then, introducing a similarity function $Q_{n}(T)$ for $U_{n}(t)$ and $\Gamma_{n}(t)$, we have

$$
\begin{gathered}
U_{n}(t)=Q_{n}\left(t / \tau_{n}^{(u)}\right)=Q_{n}(T), \\
\Gamma_{n}(t) / \Gamma_{n}(t=0)=Q_{n}\left(t / \tau_{n}^{(\gamma)}\right)=Q_{n}\left(T / \widetilde{\tau}_{n}\right) .
\end{gathered}
$$

Therefore, the similarity function is given by

$$
Q_{n}(T)=U_{n}\left(T \tau_{n}^{(u)}\right)=\frac{\Gamma_{n}\left(T \tau_{n}^{(\gamma)}\right)}{\Gamma_{n}(t=0)} .
$$

Using Eq. (2.14) for $\Gamma_{n}(t=0)$ in Eq. (4.3) leads to

$$
\Gamma_{n}(t)=\frac{1}{\tau_{n}^{(u)} \tau_{n}^{(\gamma)}} Q_{n}\left(T / \widetilde{\tau}_{n}\right) .
$$

Therefore, inserting Eqs. (4.2) and (4.5) into Eq. (2.7), we obtain

$$
\frac{d}{d T} Q_{n}(T)=-\int_{0}^{T / \tilde{\tau}_{n}} Q_{n}(S) Q_{n}\left(T-\tilde{\tau}_{n} S\right) d S .
$$

This is the non-Markovian nonlinear evolution equation for the similarity function $Q_{n}(T)$, which is called the SA equa- 

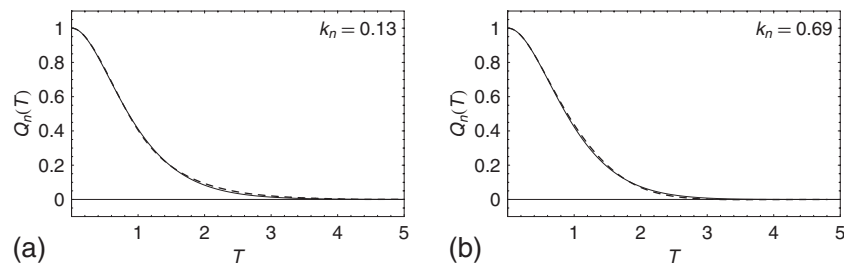

FIG. 6. Agreement of the numerical solution of the SA equation (4.6) (solid line) with the DNS of the KS equations (broken line).

tion [5]. Figure 6 shows that the numerical solution of this equation agrees with the DNS of the KS equation quite well. Thus it turns out that there exists a similarity law for $\Gamma_{n}(t)$ that enables to determine $\Gamma_{n}(t)$ explicitly.

In order to derive the algebraic decay (2.16) from the evolution equation (4.6), we consider the contribution of the deterministic short orbits to $Q_{n}(T)$ in the initial regime $T / \widetilde{\tau}_{n}=t / \tau_{n}^{(\gamma)}<1$ with $\widetilde{\tau}_{n} \leq 1$. We assume that $Q_{n}(T)$ is a simple function of $T$ with a maximum at $T=0$, just as Fig. 6 indicates. Then $Q_{n}(S) Q_{n}\left(T-\widetilde{\tau}_{n} S\right)$ in Eq. (4.6) does not change very much in a short time within the initial regime $0<S<T / \widetilde{\tau}_{n}<1$ and may be approximated by $\left\{Q_{n}(T)\right\}^{2}$, so that Eq. (4.6) may be written as

$$
d Q_{n}(T) / d T \simeq-\left\{Q_{n}(T)\right\}^{2} T / \widetilde{\tau}_{n} .
$$

This is integrated to give

$$
\frac{1}{Q_{n}(T)} \simeq \frac{T^{2}}{2 \widetilde{\tau}_{n}}+1
$$

Hence, inserting Eq. (4.8) into Eq. (4.2), we obtain

$$
U_{n}(t)=Q_{n}(T) \simeq \frac{1}{1+\left(\gamma_{n a} t\right)^{2}} \quad \text { for } t<\tau_{n}^{(\gamma)},
$$

where $\gamma_{n a}$ is given by Eq. (2.19) and $t / \tau_{n}^{(\gamma)}=T / \widetilde{\tau}_{n}<1$. Thus it turns out that the algebraic decay (2.16) can be derived from the SA equation (4.6) theoretically.

It is shown that the reversible algebraic decay (4.9) in the initial regime $t<\tau_{n}^{(\gamma)}$ also obeys a dynamic scaling law with scaling exponent $z=1$. As shown in a previous paper [4], the Fourier-Laplace transform of Eq. (4.9) leads to

$$
U_{n a}(\omega)=\frac{\pi}{2 \gamma_{n a}} \exp \left(-\frac{\omega}{\gamma_{n a}}\right)-\frac{i}{\omega}\left(1+\frac{2 \gamma_{n a}^{2}}{\omega^{2}}\right)
$$

for $\omega \gg \gamma_{n a}$, where we have added the imaginary part $U_{n a}^{\prime \prime}(\omega)$ derived previously [4]. According to Fig. 7, we have

$$
\gamma_{n a}=\mu k_{n} \quad(\mu=0.65, z=1)
$$

for $k_{n}<0.15$. Therefore, Eq. (4.10) takes the form

$$
U_{n a}(\omega)=k_{n}^{-1} \phi\left(\omega / k_{n}\right),
$$

where we have defined

$$
\phi(\eta) \equiv \frac{\pi}{2 \mu} \exp (-\eta / \mu)-\frac{i}{\eta}\left(1+\frac{2 \mu^{2}}{\eta^{2}}\right)
$$

for $\eta \equiv \omega / k_{n} \gg \mu$. Thus it turns out that the dynamic structure (4.10) obeys the dynamic scaling law (4.12) with scaling

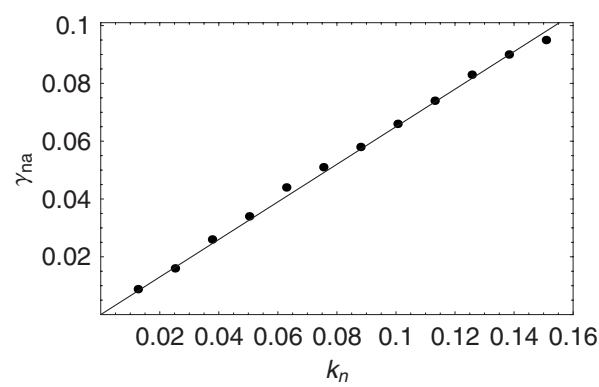

FIG. 7. The $k_{n}$ dependence of $\gamma_{n a}$ with the solid line, showing Eq. (4.11).

exponent $z=1$. The memory spectrum $\Gamma_{n}(\omega)$ can be obtained from Eq. (3.9) by inserting Eq. (4.12) into $U_{n}(\omega)$. Then we have

$$
\Gamma_{n a}(\omega)=-i \omega+\frac{k_{n}}{\phi\left(\omega / k_{n}\right)}=k_{n} \psi\left(\omega / k_{n}\right),
$$

where we have defined

$$
\psi(\eta) \equiv-i \eta+\frac{1}{\frac{\pi}{2 \mu} \exp (-\eta / \mu)-\frac{i}{\eta}\left(1+\frac{2 \mu^{2}}{\eta^{2}}\right)}
$$

for $\eta \equiv \omega / k_{n} \gg \mu$. Thus it turns out that the dynamic structures (4.12) and (4.14), produced by the dynamical coherence in the initial regime $t<\tau_{n}^{(\gamma)}$, obey the dynamic scaling law with scaling exponent $z=1$.

The basic decay rates (3.5) and (4.11) of the dynamic scaling laws are also summarized in Table I.

\section{POWER-LAW DECAY $t^{-3 / 2}$ IN CASE OF $\tau_{n}^{(\gamma)} \approx \tau_{n}^{(u)}$}

Let us consider the Fourier-Laplace transform of the similarity function (4.4), namely,

$$
Q_{n}(\Omega) \equiv \int_{0}^{\infty} Q_{n}(T) e^{-i \Omega T} d T
$$

with $\Omega T=\omega t, \Omega \equiv \omega \tau_{n}^{(u)}$, and $Q_{n}(\Omega)=U_{n}(\omega) / \tau_{n}^{(u)}$. Then the SA equation (4.6) takes the form

$$
Q_{n}(\Omega) Q_{n}\left(\widetilde{\tau}_{n} \Omega\right)+i \Omega Q_{n}(\Omega)-1=0 .
$$

In Fig. 1, let us assume that the wave number $k_{n}$ is large enough to give

$$
\widetilde{\tau}_{n}=\tau_{n}^{(\gamma)} / \tau_{n}^{(u)} \approx 1 .
$$

Then Eq. (5.2) becomes $Q_{n}^{2}+i \Omega Q_{n}-1=0$, whose solution gives the following real part $Q_{n}^{\prime}(\Omega)$ of $Q_{n}(\Omega)$ :

$$
Q_{n}^{\prime}(\Omega)= \begin{cases}\left\{1-(\Omega / 2)^{2}\right\}^{1 / 2} & \text { for } 0 \leq \Omega \leq 2 \\ 0 & \text { for } \Omega>2 .\end{cases}
$$

Hence the inverse transform of Eq. (5.1) leads to

$$
Q_{n}(T)=\frac{2}{T} \int_{0}^{\infty} Q_{n}^{\prime}(\Omega) \cos (\Omega T) d \Omega=J_{1}(2 T) / T,
$$

where $J_{1}(x)$ is the Bessel function of the first kind. For large $T \gg 1$, this takes the form 


$$
Q_{n}(T)=\frac{1}{\sqrt{\pi}} T^{-3 / 2} \cos \left(2 T-\frac{3 \pi}{4}\right) .
$$

This means that the time correlation (4.2) obeys the powerlaw decay $t^{-3 / 2}$ for $t \gg \tau_{n}^{(\gamma)}$.

This is in a strong contrast to the initial regime $t<\tau_{n}^{(\gamma)}$, where the algebraic decay (2.16) or (4.9) holds even if $\tau_{n}^{(\gamma)}$ $=\tau_{n}^{(u)}$, as shown in Sec. IV.

\section{FOUR LOCAL FORMS OF THE POWER SPECTRA}

The power spectrum $I_{n}(\omega)$ of the time series $u_{n}(t)$ is related to the time correlation function $(2.5)$ by the WienerKhintchine theorem [9] as follows:

$$
\begin{aligned}
I_{n}(\omega) \equiv \lim _{\tau \rightarrow \infty} \frac{\tau}{2 \pi}\left\langle\left|\frac{1}{\tau} \int_{0}^{\tau} u_{n}(t) e^{-i \omega t} d t\right|^{2}\right\rangle \\
=(1 / \pi)\left\langle\left|u_{n}(0)\right|^{2}\right\rangle U_{n}^{\prime}(\omega),
\end{aligned}
$$

where $U_{n}^{\prime}(\omega)$ is the real part of the Fourier-Laplace transform (2.9),

$$
U_{n}(\omega) \equiv \int_{0}^{\infty} U_{n}(t) e^{-i \omega t} d t=U_{n}^{\prime}(\omega)+i U_{n}^{\prime \prime}(\omega) .
$$

First let us consider the small wave number regime (2.15), so that we have $\tau_{n}^{(\gamma)} \ll \tau_{n}^{(u)}$. Then inserting Eqs. (2.17) and (2.16) into $U_{n}(t)$ in Eq. (6.3), we obtain

$$
\begin{gathered}
U_{n}^{\prime}(\omega)=\frac{\alpha_{n e}}{\gamma_{n e}} \frac{1}{1+\left(\omega / \gamma_{n e}\right)^{2}} \quad \text { for } \omega<1 / \tau_{n}^{(\gamma)}, \\
U_{n}^{\prime}(\omega)=\frac{\pi}{2 \gamma_{n a}} \exp \left(-\frac{\omega}{\gamma_{n a}}\right) \quad \text { for } \omega>1 / \tau_{n}^{(\gamma)} .
\end{gathered}
$$

Therefore, inserting Eqs. (6.4) and (6.5) into Eq. (6.2), we obtain a Lorentzian spectrum which consists of a Lorentzian peak (6.4) around $\omega=0$ with a linewidth $\gamma_{n e}$ and an exponential wing (6.5). This is consistent with (3.7) and (4.10) and agrees with the previous results [4].

Next let us consider the smaller wave number regime $k_{n}$ $<0.04$, so that we have $\gamma_{n e}=\lambda k_{n}^{2}$ and $\alpha_{n e} \approx 1$. Then we have dynamic scaling laws (3.13) and (4.12), which lead to

$$
\begin{gathered}
U_{n}^{\prime}(\omega)=k_{n}^{-2} \frac{\lambda}{\lambda^{2}+\left(\omega / k_{n}^{2}\right)^{2}} \quad \text { for } \omega<1 / \tau_{n}^{(\gamma)}, \\
U_{n}^{\prime}(\omega)=\frac{\pi}{2 \mu k_{n}} \exp \left(-\frac{\omega}{\mu k_{n}}\right) \quad \text { for } \omega>1 / \tau_{n}^{(\gamma)} .
\end{gathered}
$$

These dependences on $k_{n}$ and $\omega$ give the dynamic structures of the power spectra in the large-distance regime with $k_{n}$ $<0.04$.

We now increase $k_{n}$ beyond its value 0.15 , so that the Markovian condition (2.15) breaks down. Then it has been found recently [5] that, in the case of

$$
0.37 \leq \tau_{n}^{(\gamma)} / \tau_{n}^{(u)} \leq 0.84
$$

$U_{n}(t)$ takes the decay form

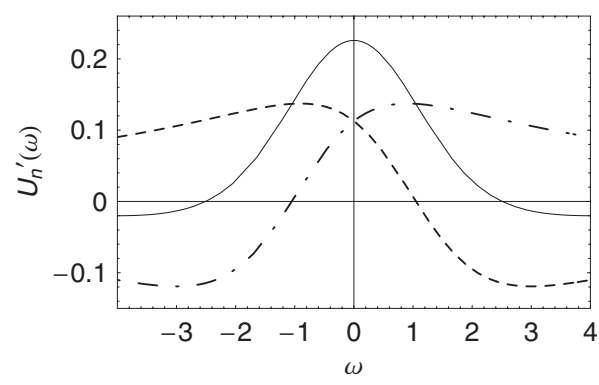

FIG. 8. The real part of the time correlation spectrum $U_{n}^{\prime}(\omega)$ for $\omega_{n}=0.91, \gamma_{n e}=1.95, \theta_{n}=-1.5$, and $\alpha_{n e}=1:-, U_{n}^{\prime}(\omega) ;-\cdot-, U_{n}^{(+)}(\omega)$; and ---, $U_{n}^{(-)}(\omega)$.

$$
U_{n}(t)=\alpha_{n e} \exp \left(-\gamma_{n e} t\right) \cos \left(\omega_{n} t+\theta_{n}\right)
$$

for $t>\tau_{n}^{(\gamma)}$. Then it is well known that the power spectrum (6.2) takes the asymmetric Lorentzian peak,

$$
\begin{gathered}
U_{n}^{\prime}(\omega)=U_{n}^{(+)}(\omega)+U_{n}^{(-)}(\omega), \\
U_{n}^{(+)}(\omega) \equiv \frac{\alpha_{n e}}{2} \frac{\left(\omega-\omega_{n}\right) \sin \theta_{n}+\gamma_{n e} \cos \theta_{n}}{\left(\omega-\omega_{n}\right)^{2}+\gamma_{n e}^{2}}, \\
U_{n}^{(-)}(\omega) \equiv \frac{\alpha_{n e}}{2} \frac{-\left(\omega+\omega_{n}\right) \sin \theta_{n}+\gamma_{n e} \cos \theta_{n}}{\left(\omega+\omega_{n}\right)^{2}+\gamma_{n e}^{2}}
\end{gathered}
$$

around $\omega=\omega_{n}$. This is a generalization of Eq. (6.4). The sharp asymmetric Lorentzian peak, however, does not appear in the case of the KS equation because $U_{n}^{\prime}(\omega)$ consists of three curves of Fig. 8 which are not sharp peaks.

In the case of $\tau_{n}^{(\gamma)} \approx \tau_{n}^{(u)}$, Eq. (5.4) with $\Omega=\omega \tau_{n}^{(u)}$ gives the power spectrum

$$
U_{n}^{\prime}(\omega)=\tau_{n}^{(u)} Q_{n}^{\prime}(\Omega)=\tau_{n}^{(u)}\left\{1-\left(\tau_{n}^{(u)} \omega / 2\right)^{2}\right\}^{1 / 2} .
$$

In the neighborhood of $\omega=0$, we have

$$
\left\{1-\left(\frac{\tau_{n}^{(u)} \omega}{2}\right)^{2}\right\}^{1 / 2} \simeq \frac{1}{1+\frac{1}{2}\left(\tau_{n}^{(u)} \omega / 2\right)^{2}}
$$

This leads to the power spectrum

$$
U_{n}^{\prime}(\omega) \simeq \frac{2 \sqrt{2} \tilde{\gamma}_{n e}}{\omega^{2}+\widetilde{\gamma}_{n e}^{2}}
$$

where we have defined $\tilde{\gamma}_{n e} \equiv 2 \sqrt{2} / \tau_{n}^{(u)}$. Therefore, although Eq. (5.4) leads to the power-law decay (5.6), its power spectrum (6.15) exhibits a Lorentzian peak with a linewidth $\widetilde{\gamma}_{n e}$.

Thus it turns out that, although the two local forms (6.4) and (6.15) give symmetric Lorentzian peaks, the local form (6.10) brings about a round hill like Fig. 8, and the local form (6.5) gives an exponential wing.

\section{SUMMARY}

A statistical mechanical theory of chaos and turbulence has been developed by exploring the time correlation function $U_{n}(t)$ defined by Eq. (2.5) and its memory function $\Gamma_{n}(t)$ 
given by Eqs. (2.8) and (3.9) for the chaotic KS equation [4-6]. Their time scales $\tau_{n}^{(u)}$ and $\tau_{n}^{(\gamma)}$, defined by Eqs. (2.11) and (2.12), and their dependence on the wave number $k_{n}$, shown in Fig. 1, give a fundamental information on the dynamic structure of the spectra $U_{n}(\omega)$ and $\Gamma_{n}(\omega)$ in Eq. (2.9).

It has been shown in Secs. III and IV that if $\tau_{n}^{(\gamma)} \ll \tau_{n}^{(u)}$, then the decay form of the time correlation function $U_{n}(t)$ of chaos and turbulence consists of the algebraic decay (2.16) in the initial regime $t<\tau_{n}^{(\gamma)}$ and the exponential decay (2.17) in the final regime $t>\tau_{n}^{(\gamma)}$. Then it has turned out that if the wave number $k_{n}$ is sufficiently small, then the Fourier-Laplace transform of the exponential decay (3.7) leads to the hydrodynamic scaling law (3.13)-(3.15) with scaling exponent $z=2$. The FourierLaplace transform of the algebraic decay (4.10) in the initial regime $t<\tau_{n}^{(\gamma)}$ leads to the dynamic scaling law (4.12)-(4.15) with scaling exponent $z=1$.

It has been shown in Sec. $\mathrm{V}$ that if $\tau_{n}^{(\gamma)} \approx \tau_{n}^{(u)}$, then the decay of the time correlation (4.2) takes the form (5.6), leading to the power-law decay $t^{-3 / 2}$ for $t \gg \tau_{n}^{(\gamma)}$.

Thus it has turned out that the time correlation spectra $U_{n}(\omega)$ and the memory spectra $\Gamma_{n}(\omega)$ bring about interesting dual structures and obey two types of dynamic scaling laws with scaling exponents $z=2$ and $z=1$, hence giving the outstanding features of chaos and turbulence.

In chaotic systems of a few degrees of freedom, such as the Duffing equation [2], the Hénon-Heiles system [18,19], and the Rössler system [20], the decay time $\tau_{n}^{(\gamma)}$ of the memory function seems to be nearly equal to the decay time $\tau_{n}^{(u)}$ of the time correlation functions of the state variables, so that we do not have the exponential decay but instead we do have long-time tails, such as $t^{-\beta}(1<\beta \leq 3 / 2)$ and $\exp \left[-(\gamma t)^{\nu}\right](0<\nu<1)$. In the initial regime $t<\tau_{n}^{(\gamma)}$, however, we do have the algebraic decay,

$$
U(t)=\frac{1}{1+b t^{2}} \quad \text { or } \quad \frac{1}{1+a t+c t^{3}},
$$

as will be discussed on other occasion.

\section{ACKNOWLEDGMENTS}

It is our pleasure to thank Professor S.-I. Itoh and Professor K. Itoh for valuable discussions on turbulence and transport processes. This work was partially supported by the collaboration programs of RIAM of Kyushu University (Grant No. 21FP-9) and by the Grant-in-Aid for Scientific Research S (Grant No. 21224014) of JSPS.
[1] P. Bergé, Y. Pomeau, and C. Vidal, Order within Chaos: Towards a Deterministic Approach to Turbulence (Hermann, Paris, 1984).

[2] J. Guckenheimer and P. Holmes, Nonlinear Oscillations, Dynamical Systems, and Bifurcations of Vector Fields (SpringerVerlag, Berlin, 1983).

[3] H. Mori and Y. Kuramoto, Dissipative Structures and Chaos (Springer-Verlag, Berlin, 1998).

[4] H. Mori and M. Okamura, Phys. Rev. E 76, 061104 (2007).

[5] M. Okamura and H. Mori, Phys. Rev. E 79, 056312 (2009).

[6] K. Sneppen, J. Krug, M. H. Jensen, C. Jayaprakash, and T. Bohr, Phys. Rev. A 46, R7351 (1992).

[7] M. Okamura, Phys. Rev. E 74, 046210 (2006).

[8] H. Mori, Prog. Theor. Phys. 33, 423 (1965).

[9] R. Kubo, M. Toda, and N. Hashitsume, Statistical Physics II, Nonequilibrium Statistical Mechanics (Springer-Verlag, Berlin, 1991).

[10] D. R. Reichman and P. Charbonneau, J. Stat. Mech.: Theory
Exp. (2005) P05013.

[11] U. Balucani, M. H. Lee, and V. Tognetti, Phys. Rep. 373, 409 (2003).

[12] P. A. Davidson, Turbulence: An Introduction for Scientists and Engineers (Oxford University Press, Oxford, 2004).

[13] D. Forster, D. R. Nelson, and M. J. Stephen, Phys. Rev. A 16, 732 (1977).

[14] V. Yakhot, Phys. Rev. A 24, 642 (1981).

[15] M. Kardar, G. Parisi, and Y. C. Zhang, Phys. Rev. Lett. 56, 889 (1986).

[16] K. Ueno, H. Sakaguchi, and M. Okamura, Phys. Rev. E 71, 046138 (2005).

[17] H. Mori, S. Kuroki, H. Tominaga, R. Ishizaki, and N. Mori, Prog. Theor. Phys. 111, 635 (2004).

[18] M. Hénon and C. Heiles, Astron. J. 69, 73 (1964).

[19] M. Hénon, Commun. Math. Phys. 50, 69 (1976).

[20] O. E. Rössler, Phys. Lett. A 57, 397 (1976). 